Afferent limb failure revisited - A retrospective, international, multicentre, cohort study of delayed rapid response team calls

\title{
Tirkkonen, Joonas
}

2020-11

Tirkkonen , J , Skrifvars , M B , Tamminen , T , Parr , M J A, Hillman , K, Efendijev , I \& Aneman , A 2020 , ' Afferent limb failure revisited - A retrospective, international, multicentre, cohort study of delayed rapid response team calls ' , Resuscitation , vol. 156 , pp. 6-14 . https://doi.org/10.1016/j.res

http://hdl.handle.net/10138/338279

https://doi.org/10.1016/j.resuscitation.2020.08.117

cc_by_nc_nd

acceptedVersion

Downloaded from Helda, University of Helsinki institutional repository.

This is an electronic reprint of the original article.

This reprint may differ from the original in pagination and typographic detail.

Please cite the original version. 


\section{Afferent limb failure revisited - a retrospective, international, multicentre, cohort study of delayed rapid response team calls}

Joonas Tirkkonen, MD, PhD. Department of Intensive Care Medicine and Department of Emergency, Anaesthesia and Pain Medicine, Tampere University Hospital, Tampere, Finland and Intensive Care Unit, Liverpool Hospital, Sydney, Australia. PO Box 2000, FI-33521 Tampere, Finland. joonas.tirkkonen@tuni.fi

Markus B Skrifvars, MD, PhD, Professor. Department of Emergency Care and Services, University of Helsinki and Helsinki University Hospital, Helsinki, Finland. PO Box 22, FI-00014 Helsinki, Finland. markus.skrifvars@hus.fi

Tero Tamminen, MD. Division of Intensive Care, Department of Anaesthesiology, Intensive Care and Pain Medicine, Helsinki University and Helsinki University Hospital. PO Box 340, FI-00029, Helsinki, HUS, Finland. tero.tamminen@hus.fi

Michael M Parr, MD, Conjoint Professor. Intensive Care Unit, Liverpool Hospital, Sydney, Australia and University of New South Wales, South Western Sydney Clinical School, Sydney, Australia. Elizabeth Street, Liverpool, NSW 2170, Australia. michael.parr@health.nsw.gov.au

Ken Hillman, MD, Professor. Intensive Care Unit, Liverpool Hospital, Sydney, Australia and University of New South Wales, South Western Sydney Clinical School, Sydney, Australia. Elizabeth Street, Liverpool, NSW 2170, Australia. k.hillman@unsw.edu.au

Ilmar Efendijev, MD, PhD. Division of Intensive Care, Department of Anaesthesiology, Intensive Care and Pain Medicine, University of Helsinki and Helsinki University Hospital. Po Box 340, FI00029, Helsinki, HUS, Finland. ilmar.efendijev@ hus.fi.

Anders Aneman, MD, PhD, Conjoint Professor. Intensive Care Unit, Liverpool Hospital, Sydney, Australia; University of New South Wales, South Western Sydney Clinical School, Sydney, Australia; Macquarie University, Faculty of Medicine and Health Sciences, Sydney, Australia. Elizabeth Street, Liverpool, NSW 2170, Australia. anders.aneman@ health.nsw.gov.au

\section{Corresponding author:}

Joonas Tirkkonen, MD, PhD. Department of Intensive Care Medicine and Department of Emergency, Anaesthesia and Pain Medicine, Tampere University Hospital, Tampere, Finland and Intensive Care Unit, Liverpool Hospital, Sydney, Australia. PO Box 2000, FI-33521 Tampere, Finland. joonas.tirkkonen@tuni.fi $+3583311611$

Word count abstract: 235

Word count article: 2,944

Tables and figures: 6

Appendices: 3 
Number of references: 36

Key words: afferent limb failure; delayed activation; rapid response team; rapid response system; vital signs 


\section{ABSTRACT}

Aim: The efficiency of rapid response teams (RRTs) is decreased by delays in activation of RRT (afferent limb failure, ALF). We categorized ALF by organ systems and investigated correlations with the vital signs subsequently observed by the RRT and associations with mortality.

Methods: International, multicentre, retrospective cohort study including adult RRT patients without treatment limitations in 2017-2018 in one Australian and two Finnish tertiary hospitals.

Results: A total of 5,568 RRT patients' first RRT activations were included. In 927 patients (17\%) ALF was present within four hours before the RRT call, most commonly for respiratory criteria (419 patients, $7.5 \%)$. In 3,516 patients (63\%) overall, and in $756(82 \%)$ of ALF patients, the RRT observed abnormal vital signs upon arrival. The organ-specific ALF corresponded to the RRT observations in $52 \%$ of cases for respiratory criteria, in $60 \%$ for haemodynamic criteria, in $55 \%$ for neurological criteria and in 52\% of cases for multiple organ criteria. Only ALF for respiratory criteria was associated with increased hospital mortality (OR 1.71, 95\% CI 1.29-2.27), whereas all, except haemodynamic, criteria at the time of RRT review were associated with increased hospital mortality.

Conclusions: Vital signs were rarely normal upon RRT arrival in patients with ALF, while organspecific ALF corresponded to subsequent RRT observations in just over half of cases. Our results suggest that systems mandating timely responses to abnormal respiratory criteria in particular may have potential to improve deteriorating patient outcomes. 


\section{INTRODUCTION}

Rapid response systems (RRSs) have been associated with decreased in-hospital cardiac arrest rates and mortality. ${ }^{1}$ However, several factors that impair the effectiveness of RRSs have been identified. ${ }^{2}$ Afferent limb failure (ALF) refers to delayed RRT activation, i.e. to the failure of ward staff to activate the rapid response team (RRT) despite objective RRT activation criteria being present hours before the RRT activation is eventually made. ${ }^{3,4}$ Since 2008 , seven studies have found ALF to be associated with increased morbidity and mortality among RRT patients, although three of these studies originated from the same hospital. ${ }^{5-11} \mathrm{~A}$ few studies have investigated patient characteristics and RRT activation reasons in RRT patients with or without activation delays, ${ }^{9-11}$ but there are no published quantitative data to explain ALF beyond such dichotomized group comparisons.

Several studies have found that ward staff may not trust their observations on vital signs to be correct if the patient appears well..$^{2,12-16}$ The fear of raising 'false alarms', may lead ward staff to wait for more abnormalities in vital signs to develop or staff may doubt that the RRT criteria are associated with worse outcomes to begin with. ${ }^{2,12-16}$ Data to objectively address these potential barriers causing afferent limb failure are warranted to facilitate timely RRT activations. Furthermore, ALF was acknowledged as one of the recommended quality metrics to be reported following a recent RRS consensus conference. ${ }^{17}$

We aimed to investigate correlations of ALF as grouped by four different organ systems with the vital signs subsequently observed by the RRT upon arrival. We further investigated the independent associations between the specific organ system ALF and hospital mortality. Finally, we investigated the associations between vital signs observed by the RRT grouped by the same organ systems and hospital mortality. These combined aims could illustrate the efficiency of RRT criteria and help alleviate any reservations among ward staff to engage with the RRS. 


\section{METHODS}

\subsection{Study description}

This is a binational, multicentre, retrospective observational cohort study including one Australian and two Finnish hospitals. This study is reported using the STROBE Statement guidelines. ${ }^{18}$

\subsection{Ethics}

The Ethics Committees of the South Western Sydney Local Health District (2019/ETH12908), Tampere University Hospital (TAYS) (R18203) and Helsinki University Hospital (HUS) (HUS/1493/2019) approved the research protocol.

\subsection{Hospitals and rapid response systems}

Liverpool Hospital is a university affiliated tertiary hospital in south western Sydney. HUS Meilahti and TAYS are university affiliated tertiary referral centres in southern Finland. The three hospitals combined have 1,700 beds to accommodate 197,000 annual admissions and provide the most advanced medical care for a population of four million. For detailed hospital characteristics please see Appendix A.

Liverpool hospital pioneered the Medical Emergency in 1990. TAYS and HUS Meilahti implemented their RRTs in 2009 and 2010. The RRT services at all three hospitals operate 24/7, and the RRTs attend both IHCAs and all other in-hospital medical emergencies (Appendix A). All three hospitals have more than ten years' experience in educating and training staff to recognize and treat deteriorating patients and manage medical emergencies.

\subsection{Rapid response team calling criteria}

All hospitals used track and trigger RRT criteria for RRT activation in 2017-2018. Appendix A presents the detailed RRT activation thresholds for individual vital signs' in each hospital. The trigger thresholds for individual vital signs are otherwise comparable between the hospitals, but 
each hospital has unique lover and upper thresholds for the respiratory rate: $<5$ or $>24,<8$ or $>28$ and $<5$ or $>30$. Further, the Liverpool hospital has also upper threshold for the systolic blood pressure (> 200mmhg) whereas the two other hospital do not have upper trigger threshold at all. Some additional differences between the hospitals' strategies in identifying deteriorating patients also exist. Liverpool implemented the 'between the flags' criteria in 2010 so that lesser deviations in vital signs would prompt ward level assessment (between the flags criteria presented in Appendix A). ${ }^{19}$ TAYS made the decision to transit from track and trigger criteria to the national early warning score (NEWS) ${ }^{20}$ in 2017, but during the study period the track and trigger criteria were in place and only the educational phase regarding the NEWS was in progress.

\subsection{Definitions}

Vital signs were defined as 'normal' or 'abnormal' according to the hospitals' objective RRT activation criteria in this study (Appendix A).

A recent systematic review ${ }^{21}$ suggested that afferent limb failure (ALF) may refer both to absolute failure when the RRT is not activated at all despite the recorded presence of RRT activation criteria (although the term has not been used in point prevalence studies investigating this phenomenon), ${ }^{22-}$ 24 and to delayed RRT activations when the team activation is delayed relative to the actual recording of patient deterioration. ${ }^{5-11}$ In this study, ALF refers to a delayed RRT activation: RRT criteria (Appendix A) had been noted and recorded by ward staff at some point between 20min four hours before the RRT call was actually made. Similar to previous studies, ${ }^{5-11}$ ALF was a dichotomous variable to capture whether positive RRT criteria had been observed \& recorded during the time window or not. ALF does not include the 'ward staff worried' criterion since the presence of this subjective RRT trigger criterion cannot be reliably noted retrospectively from the patient records.

\subsection{Abbreviations}


For study purposes, ALF was grouped by four organ systems: $\mathrm{ALF}_{\text {resp }}$ (respiratory rate and $\mathrm{SpO}_{2}$ ), $\mathrm{ALF}_{\text {haemo }}$ (heart rate, systolic blood pressure), $\mathrm{ALF}_{\text {neuro }}$ (decrease in conscious state and seizures) and $\mathrm{ALF}_{\text {multi }}$ (if a patient had ALF in two or three organ systems). The presence of abnormal vital signs observed by the RRT staff upon arrival ( $\mathrm{RRT}_{\mathrm{obs}}$ ) were similarly grouped as $\mathrm{RRT}_{\text {resp }}, \mathrm{RRT}_{\text {haemo, }}$ $\mathrm{RRT}_{\text {neuro }}$ and $\mathrm{RRT}_{\text {multi. }}$

Limitation of medical therapy (LOMT) included: 'do not attempt resuscitation', 'not for intensive care', 'not for further RRT call' and 'for comfort/palliative care'.

\subsection{Data collection}

RRT activations were continuously and prospectively recorded for governance and research purposes in all three hospitals. This study investigated RRT calls between $1^{\text {st }}$ of January 2017 to $31^{\text {st }}$ of December 2018. Data on the vital signs in RRT patients were captured from the electronic nursing records in use at all three hospitals. Detailed data on patient characteristics and subsequent in-hospital outcomes were retrospectively recorded from the electronic patient records and ICU systems. Correct linkage across all datasets was confirmed by manually matching social security numbers (Finland), medical record numbers (Australia), patient names, admission dates and RRT activation dates and times. Study data were fully anonymized before any study analyses were conducted.

\subsection{Exclusion criteria}

Any RRT patients under 18 years of age, suffering a cardiac arrest, with a preceding LOMT or already admitted to the intensive care unit (ICU) were excluded. Only the first RRT call was included for patients with multiple RRT activations.

\subsection{Analyses and Statistics}


The procedures to include and analyze patients in this study followed three steps that are illustrated in Figure 1. In the first step, we identified the study cohort, retrieved data for ALF as well as RRT observations, interventions, patient characteristics and subsequent outcomes. These data are presented in Tables 1 and 2. In the second step, we investigated the correlation between ALF by organ groups to the observations made by the RRT upon arrival (Figure 3, Appendix B). In the third step, we investigated the ALF and RRT observations grouped by organ systems and the associations with hospital mortality (Figure 4).

Data are presented as percentages with counts and continuous variables are reported with medians and interquartile $\left[\mathrm{Q}_{1}-\mathrm{Q}_{3}\right]$ range. Chi-square test was used for univariate comparisons between groups. A multivariable logistic regression model using the 'enter' method was built to investigate independent associations between the categorized ALF, categorized RRT observations, and hospital mortality. Covariates for the model were factors that were a priori judged clinically relevant or acknowledged as predictors for RRT patient outcome in previous studies (age, gender, admission type, daytime RRT activation, ICU transfer, new LOMT issued by the RRT, and study hospital). ${ }^{5-11}$ The model was checked to include $>100$ outcomes per introduced variable. Odds ratios with twosided p-values $<0.05$ were considered statistically significant and are reported with $95 \%$ confidence intervals. The SPSS Statistics software (version 25 for Windows, SPSS Inc., Chicago, IL, USA) was used for all statistical analyses. 


\section{RESULTS}

\subsection{Study cohort}

Figure 2 presents the adult RRT activations during the study period with a final cohort of 5,568 RRT patients included in the analyses. Patient characteristics, ALF according to organ system, RRT activation reasons and RRT observations according to organ system are presented in Table 1 while Table 2 presents the RRT interventions and patients' hospital outcomes.

ALF was recorded in 17\% (927) of the cases, and patients with ALF were more likely to be admitted to ICU (32\% vs. $12 \%, \mathrm{p}<0.001)$ and to die during their hospitalization (19 vs. $12 \%$, $\mathrm{p}<0.001)$ compared with patients without ALF.

A total of 3,516 patients (63\%) had abnormal vital signs upon RRT arrival (RRT obs). Patients with $\mathrm{RRT}_{\text {obs }}$ where more likely to be admitted to ICU (20\% vs. $\left.7.8 \%, \mathrm{p}<0.001\right)$ and to die during their hospitalization (16 vs. 8.1\%, p<0.001) compared with patients with normal vital signs upon RRT arrival.

Appendix B presents study cohort data according to study sites.

\subsection{Afferent limb failure vs. RRT observations upon RRT arrival}

The most common ALF was $\mathrm{ALF}_{\text {resp }}$, while the most common $\mathrm{RRT}_{\text {obs }}$ was $\mathrm{RRT}_{\text {haemo }}$ (Table 1). Eighteen percent of the patients with ALF had normal vital signs upon RRT arrival. The organ system ALF correlated to same organ system RRT obs $_{\text {in }} 52-60 \%$ of the cases. Figure 3 presents the

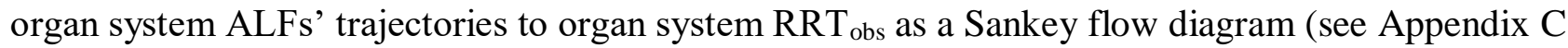
for percentages and counts). Figure 3 reads as a timeline from left to right. The left border presents the ALF grouped by four organ systems. The horizontal arrow represents the time period of $20 \mathrm{~min}-$ 4 hours preceding the RRT activations where the ALFs occurred. The right vertical arrow 
represents the RRT activation and the presence/absence of abnormal vital signs observed by the RRT staff upon arrival (RRT $\mathrm{Rbs}_{\text {) }}$.

\subsection{Associations between organ system ALF, RRT obs $_{\text {and hospital mortality }}$}

Figure 4 presents the results of the multivariable models. ALF as a dichotomized variable was associated with increased hospital mortality. When ALF was grouped by organ systems, only $\mathrm{ALF}_{\text {resp }}$ was associated with increased hospital mortality.

The presence of $\mathrm{RRT}_{\mathrm{obs}}$ as a dichotomized variable was associated with increased hospital mortality. When grouped by organ system, $\mathrm{RRT}_{\text {haemo }}$ was associated with decreased hospital mortality while all other organ systems were associated with increased hospital mortality. The

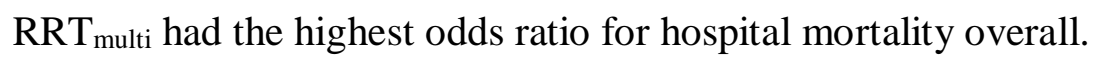




\section{DISCUSSION}

\subsection{Key findings}

This binational, multicentre study including over 5,500 adult RRT patients without preceding treatment limitations has three major findings. First, four in five patients with afferent limb failure had persistent abnormal vital signs when reviewed by the RRT. Second, only in about half of all patients was the ALF organ system the same as observed by the RRT upon arrival. Third, only $\mathrm{ALF}_{\text {resp }}$ was independently associated with hospital mortality while all abnormal vital signs observed by the RRT, except $\mathrm{RRT}_{\text {haemo, }}$ were independently associated with hospital mortality.

\subsection{Organ-specific afferent limb failure}

The first two reports on ALF and its association with unfavourable patient outcomes, each including approximately 200 patients, were published in 2008 from Austin Hospital, Australia. ${ }^{5,6}$ While this association has since been confirmed in a several other studies, ${ }^{7-11}$ no data on ALF within specific organ systems and the associations with patient outcomes have been published. This study provides insight into the progression of patients with ALF including the multiplication of abnormalities across several organ systems (Figure 3) and may thus serve to illustrate the natural history of deteriorating ward patients. We found an independent association between $\mathrm{ALF}_{\text {resp }}$ and hospital mortality. It has repeatedly been documented among different patient populations that respiratory abnormalities are associated with adverse outcomes. ${ }^{25-30}$ No other organ system ALF was significantly associated with mortality and thus the simple association of the presence or absence of ALF with mortality seems largely to be driven by respiratory abnormalities preceding RRT calls. However, the definition of ALF is based on abnormal vital signs preceding the RRT activation, ${ }^{5-11}$ but measuring vital signs on wards may be neglected or misinterpreted. ${ }^{22,31}$ All reasons for ALF are potentially harmful but it appears that prompt escalation of care is warranted when ward patients present respiratory abnormalities. 


\subsection{Vital signs upon RRT arrival}

While most RRT studies have documented the RRT trigger reasons, data on the actual vital signs upon RRT arrival are sparsely reported. ${ }^{7,32,33}$ The high fidelity $\mathrm{RRT}_{\text {obs }}$ data captured in a structured manner by an experienced team with critical care skills warrant more study. Boniatti et al. found that $30 \%$ of the RRT patients had normal vital signs upon RRT arrival. ${ }^{7}$ We have previously published two single centre trials investigating this subject and found that abnormal vital signs at the time of RRT arrival (whether classified as normal/abnormal according to hospital's 'track and trigger' criteria or the $\mathrm{NEWS}^{20}$ ) were independently associated with worse outcome. ${ }^{32,33}$ The present study confirms this observation in a large multicentre setting and contribute further specific observations across organ systems. Interestingly, $\mathrm{RRT}_{\text {haemo }}$ patients were more likely to survive than RRT patients with no abnormal vital signs. This is contrary to previous studies ${ }^{25-30}$ and we can only speculate on the reason(s) for the divergent results. It is possible that $\mathrm{RRT}_{\text {haemo }}$ identifies patients that respond particularly well to cardiovascular interventions without long-lasting implications while $\mathrm{RRT}_{\mathrm{obs}}$ in other organ-groups signify more complex pathology less amenable to therapeutic measures. It is also possible that the track and trigger criteria for haemodynamic compromise have low sensitivity and positive predictive values for detecting risk patients ${ }^{34}$ or at least lower compared to triggers for other organ systems.

\subsection{Subjective barriers for RRT activation vs. reality}

Many qualitative studies have documented how ward staff fear being criticised for triggering RRT unnecessarily and feel anxious towards the team. ${ }^{2,12-16}$ Our quantitative data do not support concerns regarding 'false' RRT calls as two thirds of all patients had abnormal vital signs documented by the RRT. Doubts about misinterpreting 'normal' vital signs as 'abnormal' seem unfounded as less than one in five patients with delayed RRT activation had normal vital signs upon RRT arrival. There is little reason to doubt the reliability of the trigger criteria ${ }^{2}$ as all except haemodynamic abnormal signs observed by the RRT arrival were independently associated with mortality. Notwithstanding, 
one in eight patients was admitted to ICU or died in hospital despite the RRT observing the vital signs to be within the normal range according to the 'track and trigger' criteria, demonstrating their limited predictive capacity. Finally, a degree of over-triage is a feature of a well-established RRS. ${ }^{35}$.

It is interesting to note that in nearly half of the RRT activations with ALF, the organ system problem did not correspond to the RRT observations upon team arrival. A Danish point-prevalence study found that in 155 general ward patients with one or more abnormal vital sign, staff were unaware of all abnormalities in 67 cases and in 20 cases staff had only noted some of the abnormalities. $^{22}$ Thus it may be that if an abnormal vital sign is observed, ward staff may neglect other vital signs or misinterpret the causality of the deterioration.

\subsection{Implications for future research}

The association of ALF with subsequent poor outcomes is established, and this study contributes to the understanding of the individual components of ALF and associations with RRT observations and mortality. Although the risk for a Hawthorne effect cannot be ignored, ${ }^{36}$ future studies of ALF could use a qualitative design interviewing ward staff immediately after the RRT review to better elucidate the reason(s) behind delayed activation. The particular importance of ALF resp and $\mathrm{RRT}_{\text {resp }}$ support that further studies are urgently needed, including using wearable monitoring technology and smart information systems to detect and escalate concerning abnormalities.

\subsection{Study strengths and limitations related to internal and external validity}

The internal validity of this study is strengthened by excluding patients with preceding treatment limitations, meticulous confirmation of data cross-linkage and adjusting mortality analyses for most confounding factors. However, we could not adjust the multivariable model for specific diagnostic groups, cumulative comorbidity and ward staffing levels. The internal validity is limited by the retrospective and observational design meaning that no causality should be inferred from the associations. Detecting ALF depends on recorded abnormal vital signs in the four hours preceding 
the call and inherent to studies of ALF, some patients might still have been missed. ${ }^{5-11}$ The study hospitals' track and trigger criteria differed in the lower and upper trigger limits for the respiratory rate. This study was not designed to capture the 'absolute ALF' when a hospitalized patient with RRT criteria present never triggers RRT review. Data relevant to this issue have been reported in point-prevalence studies. ${ }^{22-24}$

The external validity of this study is strengthened by the binational, multicentre design and the large cohort including over 5,500 RRT patients. All hospitals had mature RRS with continuous staff education, governance processes and system-development. External validity might be limited by the varying definition of the time window for ALF in the literature. ${ }^{5-11}$ Our study only included tertiary hospitals, and all utilized 'track and trigger' RRT activation criteria. The results may not be applicable in hospitals with more severe resource constraints or other systems for RRT activation.

\section{CONCLUSIONS}

The vast majority of ALF patients had abnormal vital signs when eventually reviewed by the RRT. The ALF recordings grouped by organ-system corresponded to subsequent RRT observations in just over half the cases. Respiratory abnormalities in both ALF and RRT observations almost doubled the odds for in-hospital mortality. Improved systems mandating timely responses to respiratory abnormalities in particular may have the potential to improve outcomes of deteriorating patients in hospitals.

\section{Conflict of interest statement:}

Markus Skrifvars reports speaker fees from INVOS COVIDIEN and BARD Medical (Ireland) and a research grant from GE Healthcare in 2015-2016. All other authors declare that no financial or nonfinancial conflicts of interests exist.

\section{Acknowledgements}


JT has received research grants from Instrumentarium Science Foundation and Päivikki and Sakari Sohlberg Foundation. These Foundations had no role in the study design, in the collection, analysis and interpretation of data, in the writing of the manuscript or in the decision to submit the manuscript for publication. 


\section{REFERENCES}

1. Maharaj R, Raffaele I, Wendon J. Rapid response systems: a systematic review and metaanalysis. Crit Care 2015;19:254.

2. Olsen SL, Søreide E, Hillman K, Hansen BS. Succeeding with rapid response systems - a never-ending process: A systematic review of how health-care professionals perceive facilitators and barriers within the limbs of the RRS. Resuscitation 2019;144:75-90.

3. DeVita MA, Smith GB, Adam SK, et al. "Identifying the hospitalised patient in crisis"--a consensus conference on the afferent limb of rapid response systems. Resuscitation 2010;81:375-82.

4. Sundararajan K, Flabouris A, Thompson C. Diurnal variation in the performance of rapid response systems: the role of critical care services-a review article. J Intensive Care 2016;4:15.

5. Downey AW, Quach JL, Haase M, Haase-Fielitz A, Jones D, Bellomo R. Characteristics and outcomes of patients receiving a medical emergency team review for acute change in conscious state or arrhythmias. Crit Care Med 2008;36:477-81.

6. Calzavacca P, Licari E, Tee A, et al. A prospective study of factors influencing the outcome of patients after a Medical Emergency Team review. Intensive Care Med 2008;34:2112-6.

7. Boniatti MM, Azzolini N, Viana MV, et al. Delayed medical emergency team calls and associated outcomes. Crit Care Med 2014;42:26-30.

8. Calzavacca P, Licari E, Tee A, et al. The impact of Rapid Response System on delayed emergency team activation patient characteristics and outcomes-a follow-up study. Resuscitation 2010;81:31-5.

9. Tirkkonen J, Ylä-Mattila J, Olkkola KT, Huhtala H, Tenhunen J, Hoppu S. Factors associated with delayed activation of medical emergency team and excess mortality: an Utstein-style analysis. Resuscitation 2013;84:173-8. 
10. Barwise A, Thongprayoon C, Gajic O, Jensen J, Herasevich V, Pickering BW. Delayed Rapid Response Team Activation Is Associated With Increased Hospital Mortality, Morbidity, and Length of Stay in a Tertiary Care Institution. Crit Care Med 2016;44:54-63.

11. Reardon PM, Fernando SM, Murphy K, Rosenberg E, Kyeremanteng K. Factors associated with delayed rapid response team activation. J Crit Care 2018;46:73-8.

12. Massey D, Chaboyer W, Aitken L. Nurses' perceptions of accessing a Medical Emergency Team: a qualitative study. Aust Crit Care 2014;27:133-8.

13. Astroth KS, Woith WM, Stapleton SJ, Degitz RJ, Jenkins SH. Qualitative exploration of nurses' decisions to activate rapid response teams. J Clin Nurs 2013;22:2876-82.

14. Stewart J, Carman M, Spegman A, Sabol VK. Evaluation of the effect of the modified early warning system on the nurse-led activation of the rapid response system. J Nurs Care Qual 2014;29:223-9.

15. Petersen JA, Rasmussen LS, Rydahl-Hansen S. Barriers and facilitating factors related to use of early warning score among acute care nurses: a qualitative study. BMC Emerg Med $2017 ; 17: 36$.

16. McGaughey J, O'Halloran P, Porter S, Blackwood B. Early warning systems and rapid response to the deteriorating patient in hospital: A systematic realist review. J Adv Nurs 2017;73:2877-91.

17. Subbe CP, Bannard-Smith J, Bunch J, et al. Quality metrics for the evaluation of Rapid Response Systems: Proceedings from the third international consensus conference on Rapid Response Systems. Resuscitation 2019;141:1-12.

18. Von Elm E, Altman DG, Egger M, et al. The Strengthening the Reporting of Observational Studies in Epidemiology (STROBE) statement: guidelines for reporting observational studies. Lancet 2007;370:1453-7. 
19. Aneman A, Frost SA, Parr MJ, Hillman KM. Characteristics and outcomes of patients admitted to ICU following activation of the medical emergency team: impact of introducing a two-tier response system. Crit Care Med 2015;43:765-73.

20. The Royal College of Physicians. National Early Warning Score (NEWS) 2: Standardising the assessment of acute-illness severity in the NHS. London: RCP; 2017;1-77.

21. Sundararajan K, Flabouris A, Thompson C. Diurnal variation in the performance of rapid response systems: the role of critical care services-a review article. J Intensive Care. 2016;4:15.

22. Fuhrmann L, Lippert A, Perner A, Østergaard D. Incidence, staff awareness and mortality of patients at risk on general wards. Resuscitation 2008;77:325-30.

23. Tirkkonen J, Olkkola KT, Huhtala H, Tenhunen J, Hoppu S. Medical emergency team activation: performance of conventional dichotomised criteria versus national early warning score. Acta Anaesthesiol Scand 2014;58:411-9.

24. Bucknall TK, Jones D, Bellomo R, Staples M; RESCUE Investigators. Responding to medical emergencies: system characteristics under examination (RESCUE). A prospective multi-site point prevalence study. Resuscitation 2013;84:179-83.

25. Buist M, Bernard S, Nguyen TV, Moore G, Anderson J. Association between clinically abnormal observations and subsequent in-hospital mortality: a prospective study. Resuscitation 2004;62:137-41.

26. Jacques T, Harrison GA, McLaws ML, Kilborn G. Signs of critical conditions and emergency responses (SOCCER): a model for predicting adverse events in the inpatient setting. Resuscitation 2006;69:175-83.

27. Bech CN, Brabrand M, Mikkelsen S, Lassen A. Risk factors associated with short term mortality changes over time, after arrival to the emergency department. Scand J Trauma Resusc Emerg Med 2018;26:29. 
28. Ljunggren M, Castrén M, Nordberg M, Kurland L. The association between vital signs and mortality in a retrospective cohort study of an unselected emergency department population. Scand J Trauma Resusc Emerg Med 2016;24:21.

29. Orban JC, Truc M, Kerever S, et al. Comparison of presumed cardiac and respiratory causes of out-of-hospital cardiac arrest. Resuscitation 2018;129:24-8.

30. Barfod C, Lauritzen MM, Danker JK, et al. Abnormal vital signs are strong predictors for intensive care unit admission and in-hospital mortality in adults triaged in the emergency department - a prospective cohort study. Scand J Trauma Resusc Emerg Med 2012;20:28.

31. Chen J, Hillman K, Bellomo R, et al. The impact of introducing medical emergency team system on the documentations of vital signs. Resuscitation 2009;80:35-43.

32. Tirkkonen J, Kontula T, Hoppu S. Rapid response team patients triaged to remain on ward despite deranged vital signs: missed opportunities? Acta Anaesthesiol Scand 2017;61:1278-85.

33. Tirkkonen J, Karlsson S, Skrifvars MB. National early warning score (NEWS) and the new alternative $\mathrm{SpO} 2$ scale during rapid response team reviews: a prospective observational study. Scand J Trauma Resusc Emerg Med 2019;27:111.

34. Gao H, McDonnell A, Harrison DA, et al. Systematic review and evaluation of physiological track and trigger warning systems for identifying at-risk patients on the ward. Intensive Care Med 2007;33:667-79.

35. Jones D, Bellomo R, DeVita MA. Effectiveness of the Medical Emergency Team: the importance of dose. Crit Care 2009;13:313.

36. McCarney R, Warner J, Iliffe S, van Haselen R, Griffin M, Fisher P. The Hawthorne Effect: a randomised, controlled trial. BMC Med Res Methodol 2007;7:30. 


\section{TITLES AND LEGENDS TO THE FIGURES}

\section{Figure 1.}

Title: A step-by-step presentation of the study analyses.

\section{Figure 2.}

Title: Hospitals' RRT activations with the final cohort.

Legend: RRT, rapid response team; IHCA, in-hospital cardiac arrest; ICU, intensive care unit; ER, emergency room; CPR, cardiopulmonary resuscitation; DNR, do not resuscitate order.

\section{Figure 3.}

Title: Trajectories of organ-specific afferent limb failure to organ-specific vital signs observed upon RRT arrival.

Legend: Read as a timeline from left to right. The right vertical arrow presents the RRT activation with subsequent RRT observations, whereas the horizontal arrow presents the time period of 20min4 hours preceding the RRT activations where the ALFs occurred. RRT, rapid response team; ALF, afferent limb failure; $\mathrm{ALF}_{\text {resp }}$ (respiratory rate and $\mathrm{SpO}_{2}$ ); $\mathrm{ALF}_{\text {haemo }}$ (heart rate, systolic blood pressure); $\mathrm{ALF}_{\text {neuro }}$ (decrease in conscious state and seizures); $\mathrm{ALF}_{\text {multi }}$ (if a patient had ALF on two or three organ categories). $\mathrm{RRT}_{\text {resp }}, \mathrm{RRT}_{\text {haemo, }} \mathrm{RRT}_{\text {neuro, }} \mathrm{RRT}_{\text {multi }}$ and $\mathrm{RRT}_{\text {normal }}$ represent the corresponding organ-specific observations upon RRT arrival, respectively.

\section{Figure 4.}

Legend: Odds ratios with 95\% confidence intervals of organ-specific ALF (upper chart) and organspecific vital signs upon RRT arrival (lower chart) for in-hospital mortality. Analyses are adjusted for patient age, gender, admission type (surgical/medical), daytime RRT activation (7 a.m. - 16 
p.m.), intensive care unit transfer, new limitation of medical therapy issued by the RRT and study site. ALF, afferent limb failure; RRT, rapid response team. 
Appendix B-1. Patient and rapid response team review characteristics - individual study sites.

TAYS HUS Meilahti Liverpool

Patient characteristics

Age (years; median, $\mathrm{Q}_{1}-\mathrm{Q}_{3}$ )

Sex (male)

Surgical reason for admission

Time in hospital before the RRT call (days; median, $\mathrm{Q}_{1}-\mathrm{Q}_{3}$ )

RRT call at the admission date

Afferent limb failure

Respiratory

Haemodynamic

Neurological

Multiple-organ system*

Two organ system

Three organ system
$70[60,78]$

56 (493)

$59(520)$

$2[1,5]$

$22(198)$

$34(299)$

$19(70)$

$6.3(56)$

$2.3(20)$

$6.0(53)$

$5.9(52)$

0.1 (1)

$0.3(3)$
$69[55,80]$

$53(1,940)$

$33(1,204)$

$3[1,9]$

13 (426)

$5.8(215)$

1.9 (70)

$3.2(118)$

0.3 (11)

0.4 (16)

0.4 (16)

$0.0(0)$ 
RRT review characteristics

Daytime call (7 a.m. - 16 p.m.)

$43(381)$

48 (475)

$46(1,689)$

Reason for RRT activation

Respiratory criteria

$40(353)$

$31(314)$

$21(777)$

Haemodynamic criteria

$26(226)$

33 (326)

$46(1,709)$

Neurological criteria

$25(225)$

$15(152)$

$18(669)$

Nurse worried

$4.4(39)$

$15(147)$

14 (519)

Other

$5.1(45)$

$6.0(60)$

$0.2(7)$

RRT response time (min; median, $\mathrm{Q}_{1}-\mathrm{Q}_{3}$ )

$4[3,6]$

$4[3,5]$

$3[2,4]$

Abnormal vital signs upon RRT arrival

$77(672)$

$63(625)$

$60(2,219)$

Respiratory

$21(184)$

21 (209)

$13(462)$

Haemodynamic

$7.4(66)$

$16(164)$

$35(1,284)$

Neurological

$19(167)$

$9.4(94)$

$6.6(244)$

Multiple-organ system*

$29(255)$

$16(158)$

$6.1(226)$

Two organ system

$24(212)$

14 (135)

$5.6(207)$ 
Data are presented as percentages (counts). Continuous variables are reported with medians and interquartile [Q $\left.\mathrm{Q}_{1}-\mathrm{Q}_{3}\right]$ range. RRT, rapid response team. *Abnormal vital signs in two or three of the respiratory/haemodynamic/neurological systems.

Appendix B-2. Rapid response team interventions and subsequent patient outcomes - individual study sites.

TAYS HUS Meilahti Liverpool

Rapid response team interventions

\begin{tabular}{|c|c|c|c|}
\hline Supplementary oxygen & $22(192)$ & $24(240)$ & $33(1,224)$ \\
\hline CPAP/NIV & $12(103)$ & $5.6(56)$ & $6.3(231)$ \\
\hline Intravenous fluids & $22(191)$ & $25(252)$ & $38(1,390)$ \\
\hline Blood products & $6.3(56)$ & $3.0(30)$ & Data not available \\
\hline Medications & $31(279)$ & $36(359)$ & $50(1,857)$ \\
\hline Endotracheal intubation & $2.3(22)$ & $2.2(22)$ & $0.9(33)$ \\
\hline Tracheostomy care & $2.5(22)$ & $0.8(8)$ & $0.3(10)$ \\
\hline
\end{tabular}

Patient outcomes 


\begin{tabular}{|c|c|c|c|}
\hline Immediate outcome death & $0.3(3)$ & $0.3(3)$ & $0.1(5)$ \\
\hline New LOMT issued by the RRT & $7.5(67)$ & $8.5(85)$ & $1.5(54)$ \\
\hline Transfer to ICU & $20(174)$ & $35(345)$ & $9.6(355)$ \\
\hline ICU length of stay (days; median, $\mathrm{Q}_{1}-\mathrm{Q}_{3}$ ) & $2[2,4]$ & $2[1,5]$ & $2[2,5]$ \\
\hline ICU mortality & $9.8(17)$ & $8.1(28)$ & $11(40)$ \\
\hline Discharged alive but with LOMT & $13(22)$ & $5.8(20)$ & $8.5(30)$ \\
\hline Discharged alive and without any LOMT & $78(135)$ & $86(297)$ & $80(285)$ \\
\hline Hospital mortality & $15(136)$ & $11(106)$ & $13(495)$ \\
\hline
\end{tabular}

Data are presented as percentages (counts). Continuous variables are reported with medians and interquartile $\left[\mathrm{Q}_{1}-\mathrm{Q}_{3}\right]$ range. CPAP, continuous positive airway pressure; NIV, non-invasive ventilation; LOMT, limitation of medical therapy; RRT, rapid response team; ICU, intensive care unit. 
Appendix C. Visual data from Figure 3 presented as percentages (counts) in a Table.

Afferent limb failure (count)

\begin{tabular}{lllll}
\hline Any ALF (927) & ALF $_{\text {resp }}$ (419) & ALF $_{\text {haemo }}$ (293) & ALF $_{\text {neuro }}$ (87) & multi (128)
\end{tabular}

Vital signs observed upon RRT arrival by

the team

\begin{tabular}{|c|c|c|c|c|c|}
\hline $\mathrm{RRT}_{\text {normal }}$ & $18(171)$ & $18(77)$ & $20(59)$ & $16(14)$ & $16(21)$ \\
\hline $\mathrm{RRT}_{\text {resp }}$ & $26(243)$ & $52(218)$ & $2.4(7)$ & $1.1(1)$ & 13 (17) \\
\hline $\mathrm{RRT}_{\text {haemo }}$ & $21(195)$ & $1.4(6)$ & $60(175)$ & $1.1(1)$ & $10(13)$ \\
\hline $\mathrm{RRT}_{\text {neuro }}$ & $9.5(88)$ & 3.8 (16) & 4.8 (14) & $55(48)$ & $7.8(10)$ \\
\hline $\mathrm{RRT}_{\text {multi }}$ & $25(230)$ & $24(102)$ & $13(38)$ & $26(23)$ & $52(67)$ \\
\hline
\end{tabular}

Data are presented as percentages (counts). RRT, rapid response team. Data presenting cross-linking organ categories in bold. 
Table 1. Patient and rapid response team review characteristics

Patient characteristics $\%$

\begin{tabular}{llc}
\hline Age (years; median, $\left.\mathrm{Q}_{1}-\mathrm{Q}_{3}\right)$ & $69[56-79]$ & 2,996 \\
Sex (male) & 56 & 2,242 \\
Surgical reason for admission & 44 & 802 \\
Time in hospital before the RRT call (days; median, $\left.\mathrm{Q}_{1}-\mathrm{Q}_{3}\right)$ & $3[1-7]$ & 927 \\
RRT call at the admission date & 14 & 419 \\
Afferent limb failure & 17 & 293 \\
$\quad$ Respiratory & 7.5 & 87 \\
$\quad$ Haemodynamic & 5.3 & 128 \\
Neurological & 1.6 & 124 \\
Multiple-organ system* & 2.3 & 4 \\
$\quad$ Two organ system & 2.2 & 0.1 \\
Three organ system & &
\end{tabular}

RRT review characteristics

Daytime call (7 a.m. - 16 p.m.) 46

Reason for RRT activation

Respiratory criteria 26

Haemodynamic criteria

41

2,261

Neurological criteria

19

1,046

Nurse worried

13

705

Other

2.0

112

RRT response time (min; median, $\mathrm{Q}_{1}-\mathrm{Q}_{3}$ )

$3[2-5]$ 


\begin{tabular}{lcc}
\hline Abnormal vital signs upon RRT arrival & 63 & 3,516 \\
Respiratory & 15 & 855 \\
Haemodynamic & 27 & 1,517 \\
Neurological & 9.1 & 505 \\
Multiple-organ system* & 12 & 639 \\
Two organ system & 9.9 & 554 \\
Three organ system & 1.5 & 85 \\
\hline
\end{tabular}

Data are presented as percentages and numbers. Continuous variables are reported with medians and interquartile $\left[\mathrm{Q}_{1}-\mathrm{Q}_{3}\right]$ range. $\mathrm{RRT}$, rapid response team. *Abnormal vital signs in two or three of the respiratory/haemodynamic/neurological systems. 
Table 2. Rapid response team interventions and subsequent patient outcomes

\begin{tabular}{lcc}
\hline Rapid response team interventions & $\%$ & $n$ \\
\hline Supplementary oxygen & 30 & 1,656 \\
CPAP/NIV & 7.0 & 390 \\
Intravenous fluids & 33 & 1,833 \\
Medications & 45 & 2,495 \\
Endotracheal intubation & 1.3 & 75 \\
Tracheostomy care & 0.7 & 40
\end{tabular}

Patient outcomes

Immediate outcome death

0.2

11

New LOMT issued by the RRT

3.7

206

Transfer to ICU

16

875

ICU length of stay (days; median, $\mathrm{Q}_{1}-\mathrm{Q}_{3}$ )

$2.5[2-5]$

ICU mortality

9.7

85

Discharged alive but with LOMT

8.2

72

Discharged alive and without any LOMT

82

718

Hospital mortality

13

737

Data are presented as percentages and counts. Continuous variables are reported with medians and interquartile $\left[\mathrm{Q}_{1}-\mathrm{Q}_{3}\right]$ range. CPAP, continuous positive airway pressure; NIV, non-invasive ventilation; LOMT, limitation of medical therapy; RRT, rapid response team; ICU, intensive care unit. 
Step 1. Data capture. RRT=Rapid Response Team. Results presented in Tables 1 and 2.

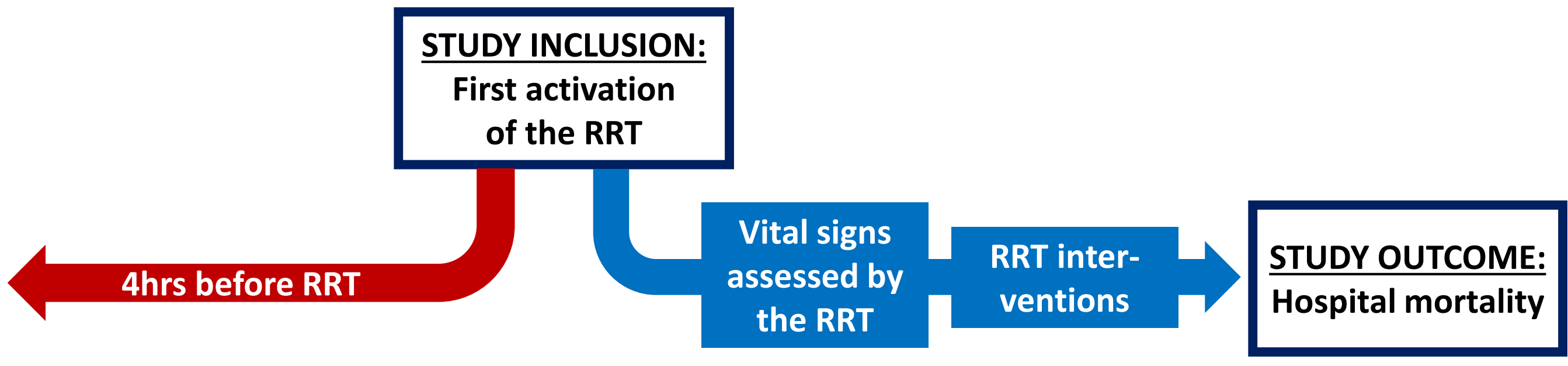

Step 2. Correlation of observations indexed by organ systems during screening for Afferent Limb Failure (ALF) and during RRT review. Results presented in Figure 3 and Appendix B.
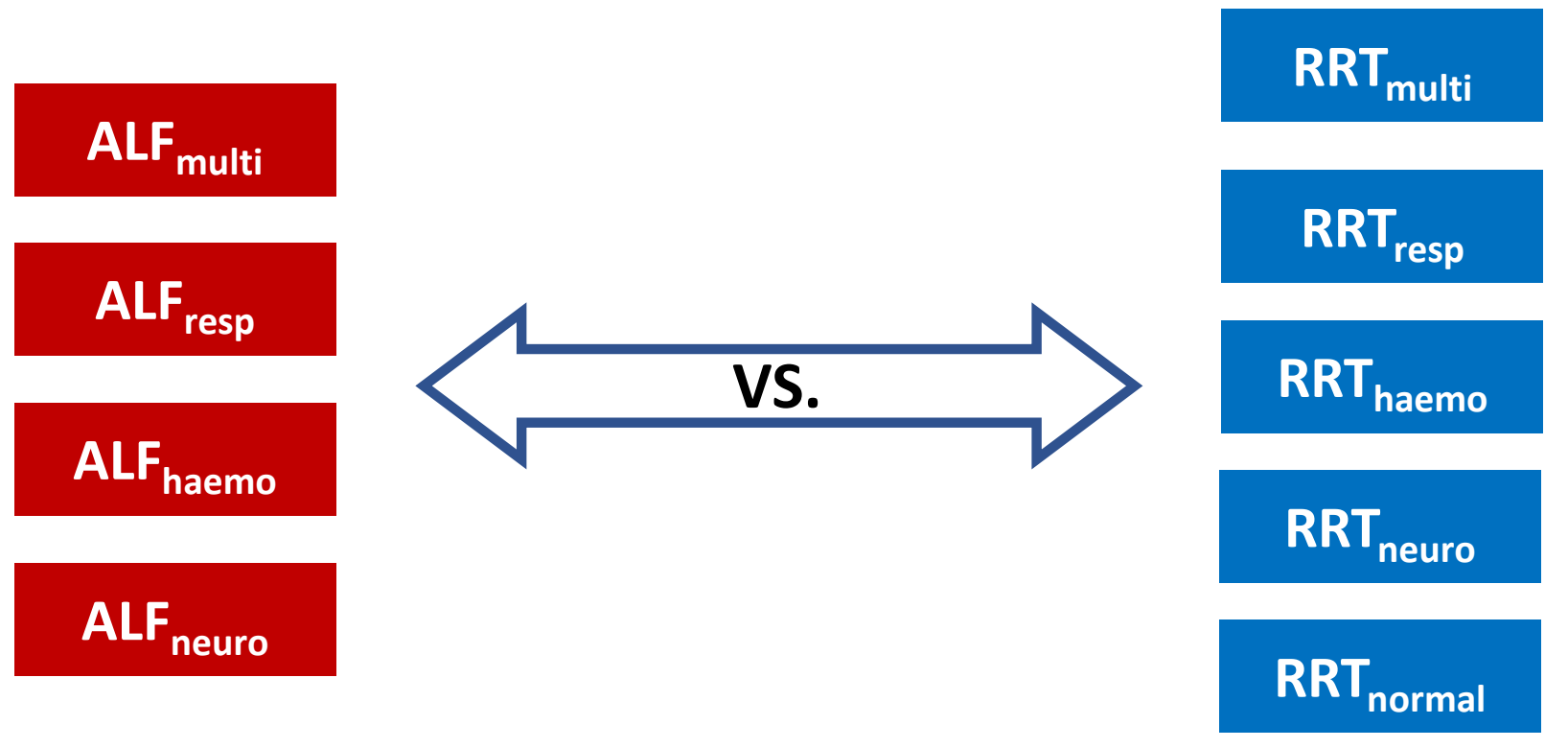

Step 3. Multivariable analysis of ALF and RRT observations indexed by organ systems vs. hospital mortality. Results presented in Figure 4.
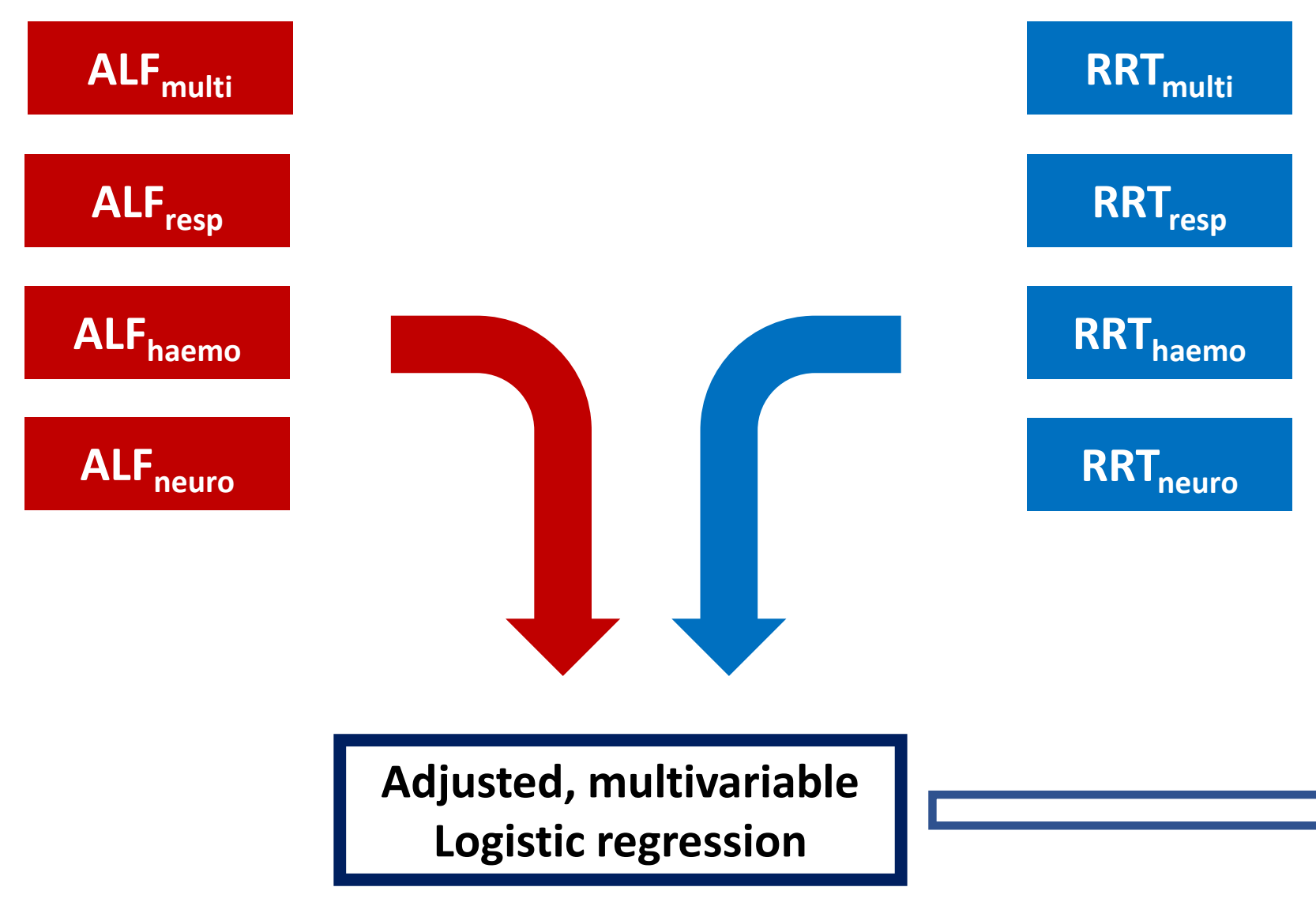
Figure 2.

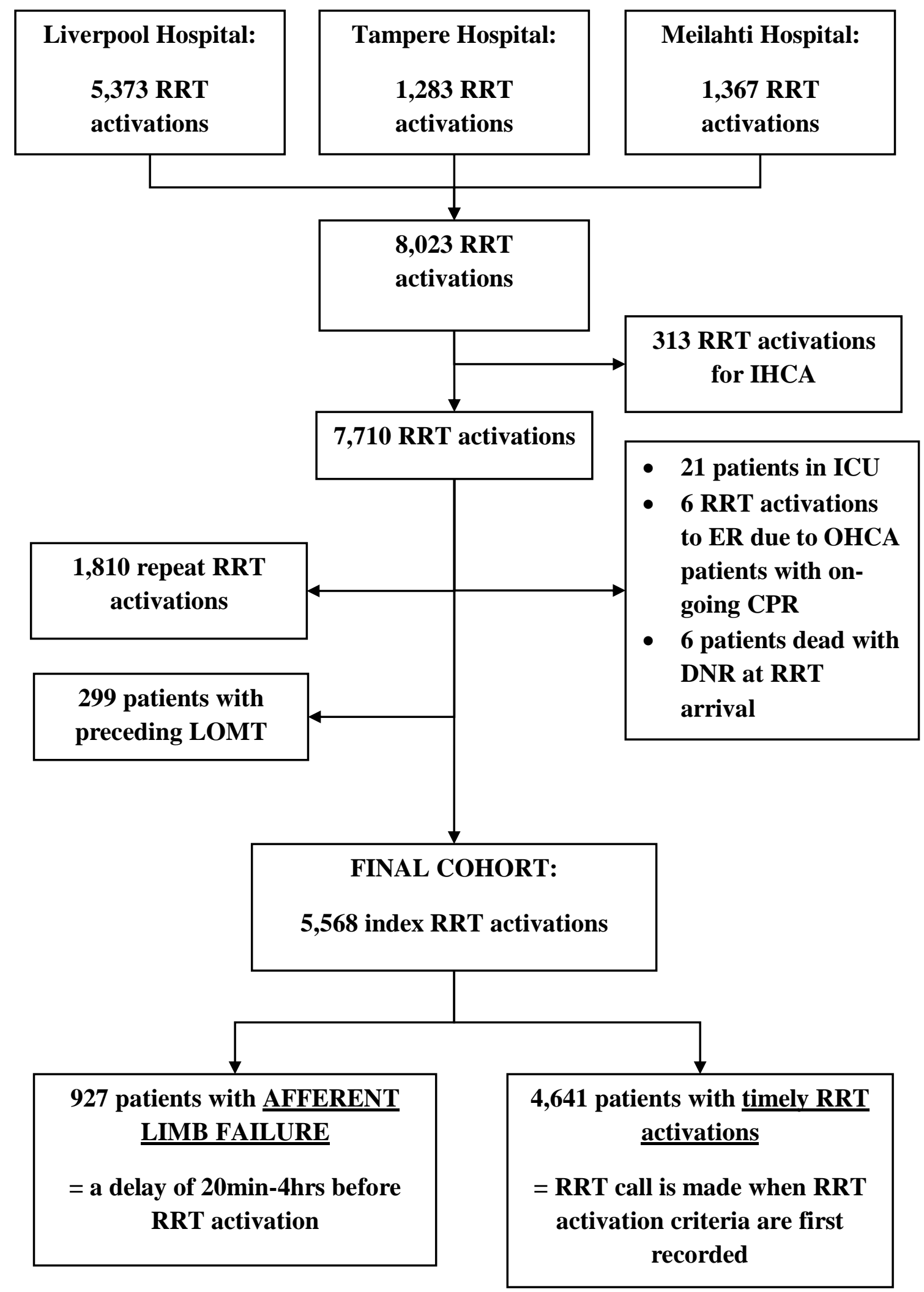








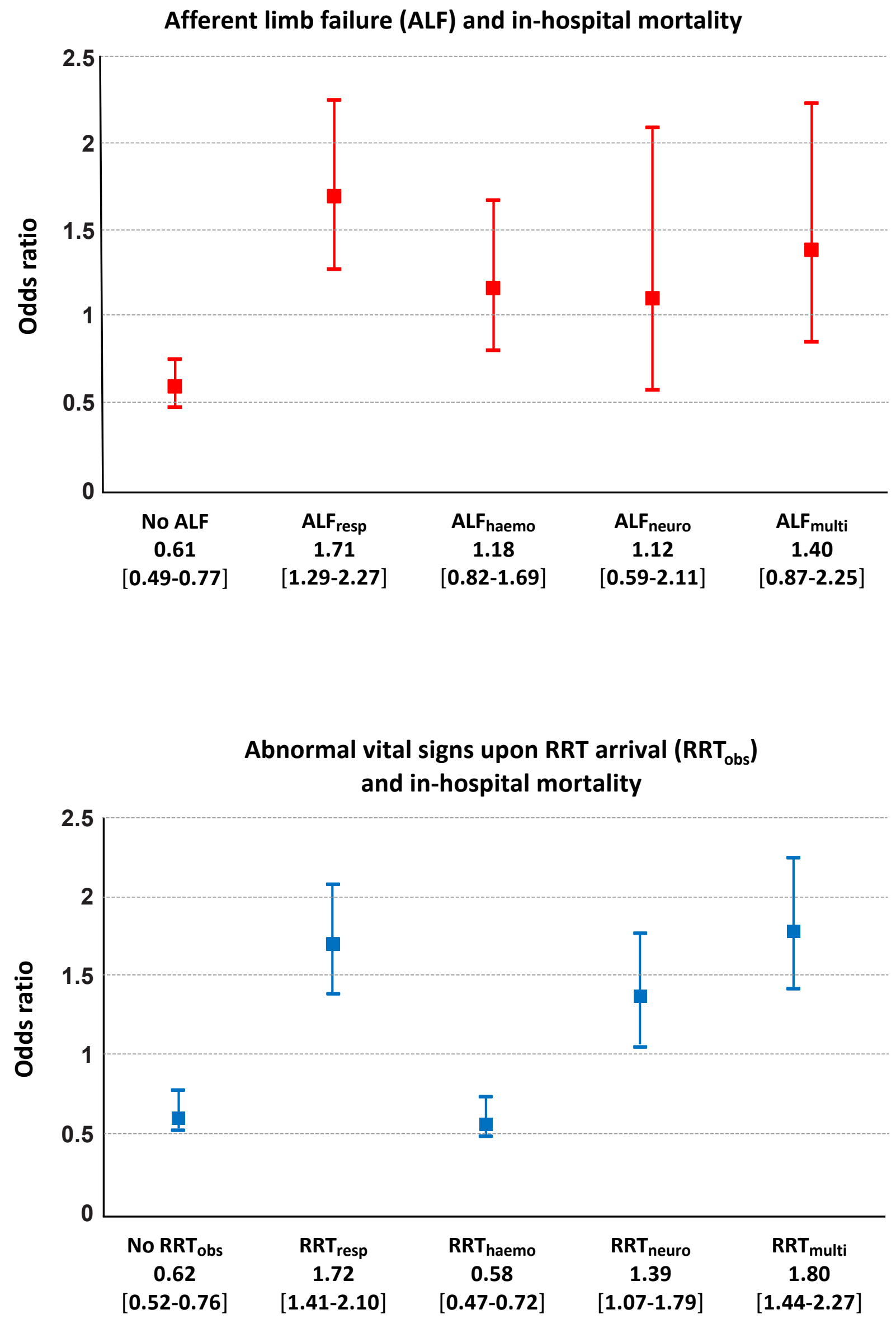